\title{
Meteorology applied to urban air pollution problems: concepts from COST 715
}

\author{
B. Fisher ${ }^{1}$, J. Kukkonen ${ }^{2}$, M. Piringer ${ }^{3}$, M. W. Rotach $^{4}$, and M. Schatzmann ${ }^{5}$ \\ ${ }^{1}$ Environment Agency, Kings Meadow House, Reading RG1 8DQ, UK \\ ${ }^{2}$ Finnish Meteorological Institute, Air Quality Research, Sahaajankatu 20 E, 00880 Helsinki, Finland \\ ${ }^{3}$ Central Institute for Meteorology and Geodynamics, Hohe Warte 38, 1190 Vienna, Austria \\ ${ }^{4}$ Swiss Federal Office for Meteorology and Climatology, MeteoSwiss, Kraebuehlstrasse 58, Zürich 8044, Switzerland \\ ${ }^{5}$ University of Hamburg Meteorological Institute, Bundesstrasse 55, 20146 Hamburg, Germany
}

Received: 13 July 2005 - Published in Atmos. Chem. Phys. Discuss.: 31 August 2005

Revised: 21 November 2005 - Accepted: 11 January 2006 - Published: 22 February 2006

\begin{abstract}
The outcome of COST 715 is reviewed from the viewpoint of a potential user who is required to consider urban meteorology within an air pollution assessment. It is shown that descriptive concepts are helpful for understanding the complex structure of the urban boundary layer, but that they only apply under a limited number of conditions. However such concepts are necessary to gain insight into both simple and complex air pollution models. It is argued that wider considerations are needed when considering routine air quality assessments involving an air quality model's formulation and pedigree. Moreover there appears to be a reluctance from model developers to move away from familiar concepts of the atmospheric boundary layer even if they are not appropriate to urban areas. An example is given from COST 715 as to how routine urban meteorological measurements of wind speed may be used and adapted for air quality assessments. Reference to the full COST 715 study is made which provides further details.
\end{abstract}

\section{Introduction}

This paper is a selective review of the COST 715 programme "Meteorology applied to Urban Air Pollution Problems" which ran between 1998-2004. COST is a European activity which supports scientific exchange and networks. In urban areas as anywhere else wind, turbulence profiles, surface heat flux and mixing height are required to run air quality pollution transport models, including both simple dispersion models and more complex, numerical simulation models (Middleton, 2002).

Correspondence to: B. Fisher

(bernard.fisher@environment-agency.gov.uk)
Meteorological pre-processing models provide the boundary conditions, or parameter values, profiles etc., needed by the pollution transport models. Such boundary conditions and parameter values and profiles are commonly supplied for rural areas. The purpose of COST 715 is to give information about methods for providing this meteorological information for urban pollution transport models. The urban situation is important, as pollution levels are generally highest in urban areas. In COST 715 a large number of methods have been reviewed. Notable results are the need to describe properly the roughness sub-layer of the atmosphere, containing and in the vicinity of large urban roughness elements, to treat the urban surface heat exchange at night, and to interpret large urban field programmes and urban air pollution episodes. In addition COST 715 provides advice on where urban meteorological data may be obtained. It suggests a so-called reference height for urban meteorological wind speed measurements. The final report of the project provides further details of the results (Fisher et al., 2005).

\section{Urban complexity and the NUSAP method of evalu- ating models}

The main feature of the urban boundary layer is its complexity. One can no longer assume that the atmospheric boundary layer is homogeneous. As seen from Fig. 1, the urban boundary layer involves wide variations in time and space scales. However at the same time decision makers require predictions relating to important issues concerning air quality and human health. In addition, some of the meteorological variables needed for urban air quality assessments are quantities that are not routinely measured, such as the surface flux parameters or the mixing layer depth. This paper considers

Published by Copernicus GmbH on behalf of the European Geosciences Union. 


\section{a) Mesoscale}

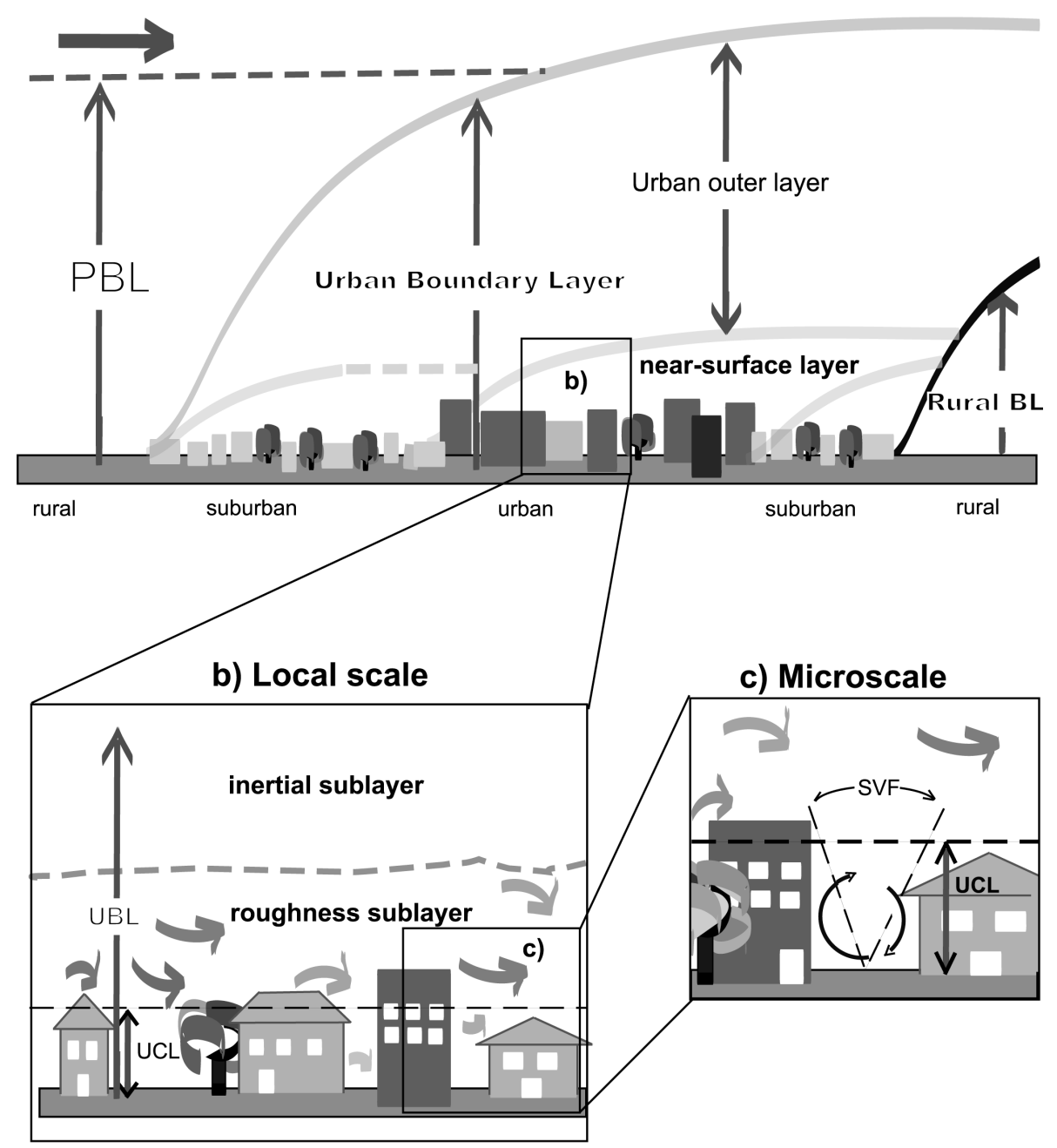

Fig. 1. Schematic diagram showing processes, flow and scale lengths within an urban boundary layer, UBL. This is set in the context of the planetary boundary layer, PBL, the urban canopy layer, UCL, and the sky view factor, SVF, a measure of the degree to which the sky is obscured by surrounding buildings at a given point which characterises the geometry of the urban canopy.

the framework for dealing with complexity, and the parallel question of uncertainty, within the urban atmospheric boundary layer. It will mainly deal with simple approaches in contrast to advanced methods. Other papers in this Special Issue of Atmospheric Chemistry and Physics, on Urban Meteorology and Atmospheric Pollution (EMS-FUMAPEX), discuss advanced models.

Modelling approaches leading to predictions and decisions have been subject to review within the so-called NUSAP framework. In this approach it is argued (Ravetz and Funtowicz, 2006 ${ }^{1}$ ) that model results should be structured to contain numbers, units, spread, assessment and pedigree. The first

\footnotetext{
${ }^{1}$ Ravetz, J. R. and Funtowicz, S. O.: The management of uncertainty and quality in quantitative information, http://www.nusap. net, 2006.
}

three are familiar to the conventional atmospheric modeller being concerned with the predictions and the uncertainty associated with predictions, though with some of the most complex models it is not possible to predict all aspects of parameter variations. This is why in the research project Integrated Systems for Forecasting Urban Meteorology, Air Pollution and Population Exposure (FUMAPEX, project website: http://fumapex.dmi.dk/, see this Special Issue) a range of models are used to introduce a spread in results.

The need for an assessment of models and consideration of their pedigree is less familiar. Assessment refers to qualitative judgement regarding the value of a model description. In this paper models of features of the urban boundary layer are described in figures, showing the characteristics of the urban flow. The modeller tries to capture these within his model. 


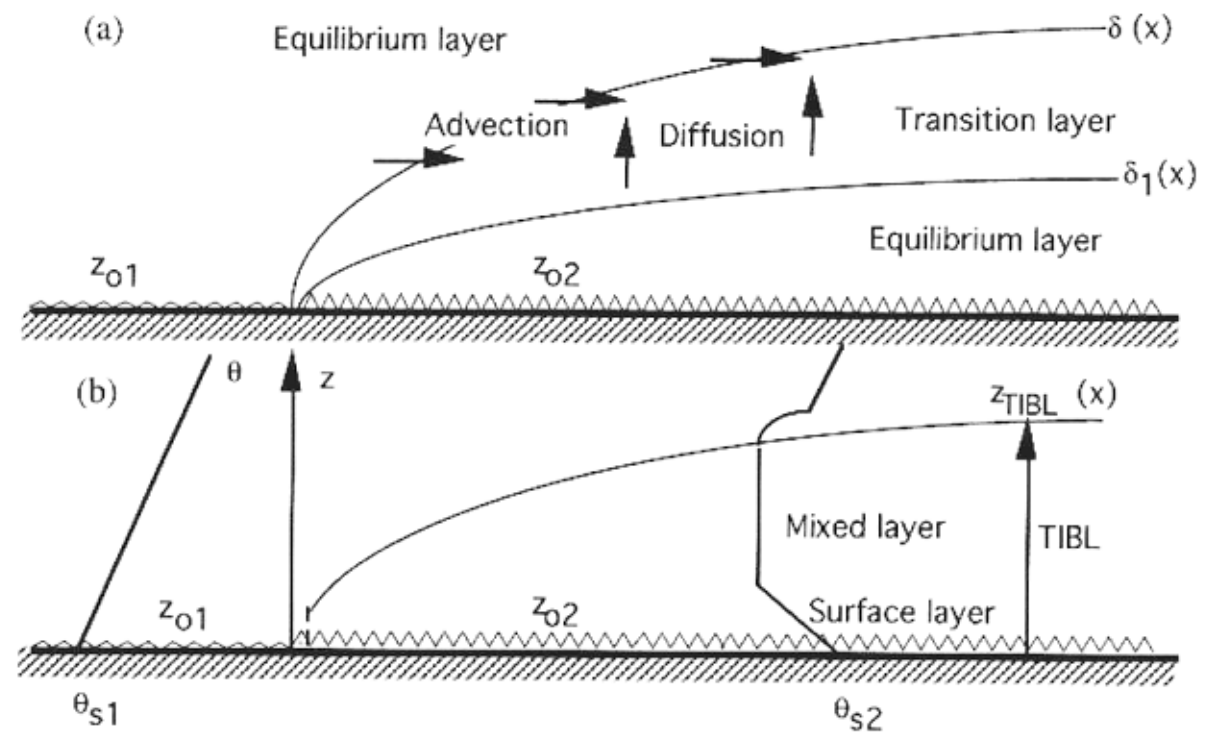

Fig. 2. Interaction of the rural boundary layer as it impacts on to the urban area showing the development of an internal boundary layer at a transition to a rougher surface $\mathrm{z}_{02} \gg \mathrm{z}_{01}$ : (a) after Hunt and Simpson, (b) after Melas and Kambezidis. The change in the potential temperature profile $\theta$ and the surface potential temperatures $\theta_{s 1}$ and $\theta_{s 2}$ are also shown, illustrating the development of the thermal internal boundary layer, TIBL.

However one cannot assume that by including these features one will necessarily obtain better predictions. An important aspect of assessment is that a model should be accessible and is not treated as a black box. Simple models are an effective way within modelling to obtain insight into how a model performs. This is why it may be advantageous to use a range of techniques in parallel e.g. numerical models, physical models and field experiments. Pedigree refers to the application of the model. A model with a good pedigree is often referred to as one which is "well established".

Assessment and pedigree also need to consider the context in which the model is used. For urban meteorology the most important decisions will apply to the Air Quality Framework Directive and to planning decisions relating to new development in a city (such as the expansion of an airport) or adding new housing to extend a city. One needs a suitable description of the meteorology in relation to the other main factors, such as emissions and the description of dispersion and chemical reactions, which affect air quality (Karppinen et al., 2000a, b). Since models are usually ultimately tested against measurements, it is not easy to tell what would be the errors arising from an incorrect treatment of meteorology. For the Air Quality Directive, exceedences of the long-term averages of $\mathrm{PM}_{10}$ and $\mathrm{NO}_{2}$ are of major interest for which quite approximate methods may be adequate. However there is also interest in episodes of high pollution, which can only be adequately covered by the application of complex mesoscale transport and pollution models.

\section{Urban dynamics}

Figure 2 shows ideas concerning the transition from a rural to an urban boundary layer in more detail. The structure is idealised to illustrate concepts. For calculating concentrations where the human exposure is highest within an urban area, the transition between the rural and urban boundary and the format of the internal urban boundary layer is not generally considered in detail. Instead the structure near the ground within the internal urban boundary layer, where the vertical exchange is in equilibrium, is the focus. The urban roughness sublayer is of much larger vertical extension than found in typical rural areas and occupies the first tens of metres above the surface, with the remainder of the surface layer (the inertial sublayer) aloft. The roughness sublayer includes the urban canopy layer, which is composed of individual street canyons and other roughness elements (see Fig. 3 which shows the layers in equilibrium). MoninObukhov similarity theory is not valid within the roughness sublayer and turbulent fluxes of momentum, energy, moisture and pollutants are height dependent. (The wind profile is sketched in Fig. 4.) The turbulent flux of momentum decreases to zero within the roughness sublayer (Rotach, 2001). This reduction is due to the drag on the flow caused by the buildings and this can be reproduced within numerical models (Martilli et al., 2002) by introducing an extra term in the momentum balance. Several approaches e.g. Soulhac et al. (2002) or Coceal and Belcher (2005), represent groups of buildings by a porous layer with a permeability, which depends on the size, orientation and spacing of the buildings. 


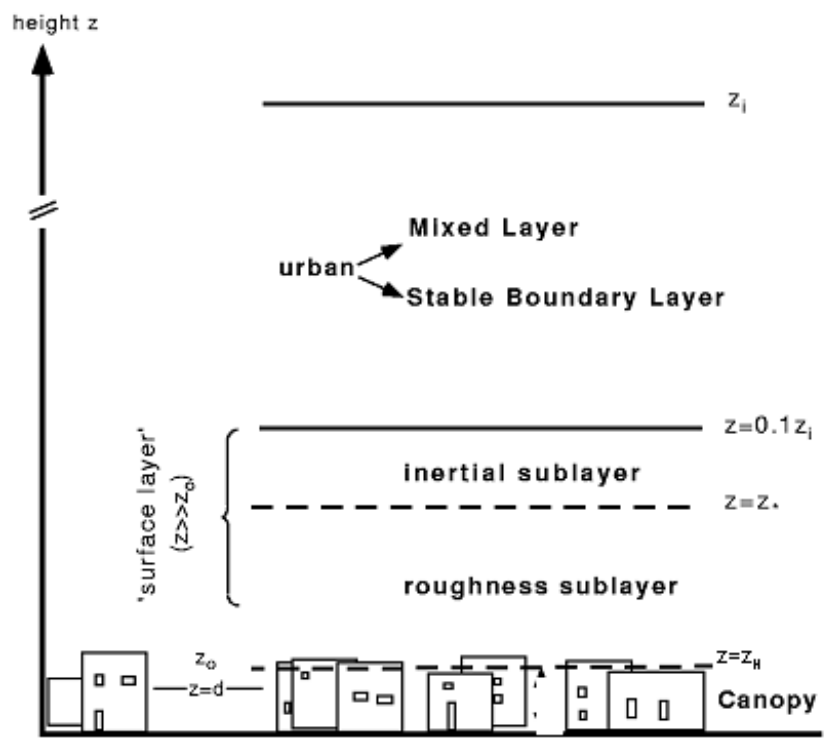

Fig. 3. Idealised structure within the equilibrium urban boundary layer in which typical heights are sketched. $z_{H}$ denotes the mean height of buildings.

Only aloft in the surface layer, are contributions from individual surface roughness elements blended into representative averages. As the building density changes in the urban area, the urban boundary layer evolves (see Fig. 2).

\section{Urban surface heat flux}

Apart from the dynamics, the surface energy balance is the key component of any model aiming to simulate dynamical and thermodynamical patterns above the surface. In its simplest one-dimensional form, it can be written as:

$Q^{*}=H+L E+G$

where $Q^{*}$ is the net all-wave radiation, $H$ and $L E$ denote turbulent sensible and latent heat fluxes, respectively, and $G$ is the storage heat flux usually not measured but determined as a residual (Piringer et al, 2002). Equation (1) is only justified if it refers to a level outside the roughness sublayer, in practical terms some 2 times above the mean building height. The "G" term then includes all the processes (advection, flux divergence etc.) in the roughness sublayer and also the anthropogenic heat. The variability in typical urban structure means that the terms on the right hand side of the heat balance equation vary within the urban area as the surface material varies. In many cities, the additional source of energy due to human activities, the anthropogenic heat flux, is significant.

In urban areas there are marked differences in energy partitioning compared to rural conditions (e.g. Christen and Vogt, 2004), where most parameterisation schemes and measurements have been performed. There is still considerable un-

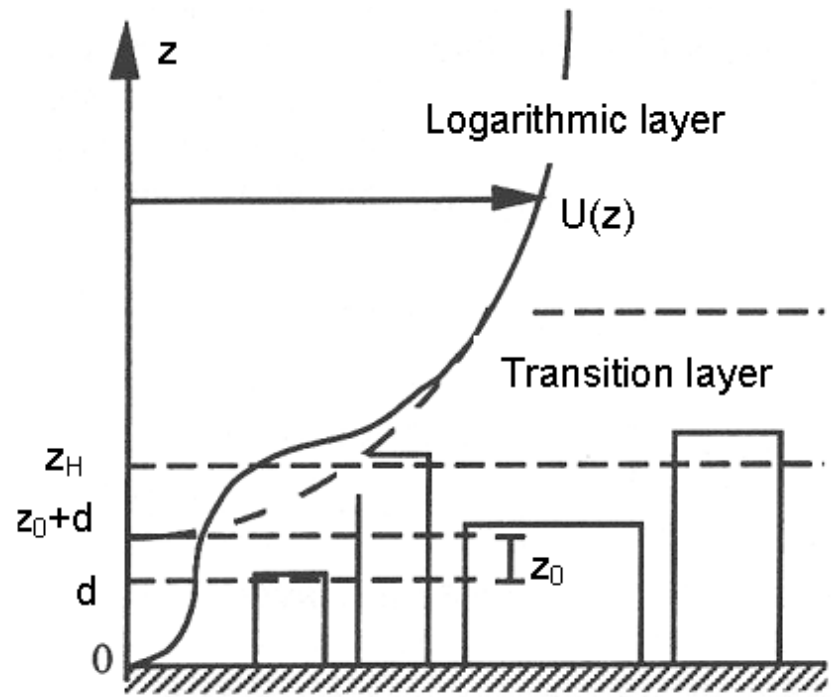

Fig. 4. Sketch of profile within the urban boundary layer. $z_{H}$ denotes the mean height of buildings.

certainty concerning the partitioning of the components of the surface energy balance and the role of surface cover (e.g. the fractions of built-up areas and green space), city surroundings, and prevailing meteorological conditions. This is reviewed in a COST report (Piringer and Joffre, 2005).

Knowledge of surface heat flux, $H$, as well as atmospheric stability and surface roughness is essential, both as input and boundary conditions, in advanced air pollution dispersion models. Normally the surface energy balance or its components $(H$ or $L E)$ are not directly measured at meteorological stations. In the last decade, a series of local-scale energy balance observations have been conducted at a restricted number of sites, largely in residential areas in North America. (See, for example, the technical programme at the American Meteorological Society Meeting in Seattle, January 2004, Symposium on Planning, Nowcasting and Forecasting in the Urban Zone, http://ams.confex.com/ams/84annual.) A focus of European research has been on surface flux measurements in recent experiments. This is explicitly concerned with testing practical schemes for estimating the surface heat flux $H$ from measured parameters, using Eq. (1) and other relations. Three new field campaigns, in Basel, Marseilles and Birmingham, studied processes in European cities. Of particular difficulty is the heat storage term $G$, so that the main atmospheric components are not in equilibrium. It is shown from experiments that night-time urban conditions remain close to neutral stability. This is why the main interest in this paper is with near neutral dynamically driven urban boundary layers.

Assessing methods to determine, or model, the height of the urban boundary layer, which is dependent on the surface heat fluxes, is another important issue (see the relevant COST 715 report, Piringer and Joffre, 2005).

In recent years, a number of boundary-layer parameterisation schemes have been developed to estimate net radiation, 
sensible heat flux and other urban boundary layer parameters from hourly standard meteorological data. An urban pre-processor scheme (LUMPS - Local-scale Urban Meteorological Pre-processing Scheme) makes use of parameterisations that require standard meteorological observations, supplemented by basic knowledge of the surface character of the target urban area. LUMPS (Grimmond and Oke, 2002) has been shown to perform well when evaluated using data from North American cities. However full urban correction factors are not widely applied in routine air pollution assessments. COST 715 conducted a review (Schatzmann et al., 2001) of current methods of obtaining urban meteorological data in Europe for pollution applications, which showed commonly applied methods are not well justified.

The concepts outlined in the next section only apply for part of the time, in part of the urban boundary layer. A full description of the urban boundary layer requires the use of numerical meso-scale transport models which usually have been used to analysis air pollution episodes (see Kukkonen et al., 2005; Rantamäki et al., 2005, and other papers in this Special Issue).

\section{General concepts regarding reduction in complexity}

The COST 715 study contains many examples of how to deal with complexity. Specifically the question is whether to define the characteristics of an urban area in terms of a few parameters? For example the basic premise of the LUMPS scheme is that heat fluxes can be modelled using net all-wave radiation, simple information on surface cover (area of vegetation, buildings and impervious materials), surface geometry (surface element roughness and density) and standard weather observations (air temperature, humidity, wind speed and pressure). The method has limited data requirements, yet is able to predict the spatial and temporal variability known to occur within urban areas. The aim is to reduce the complexity of the urban surface heat flux with a minimum increase in uncertainty. Empirical data to test the method in various urban areas is essential.

Of course there are situations where simplification does not apply. Since the LUMPS method is a one-dimensional energy balance, it is unlikely to perform well in areas where there is significant spatial variability in land cover, or surface geometry e.g. at the urban-rural transition.

The urban wind flow and the associated intensity of turbulence are the main factors determining urban concentrations. The roughness structure influences the wind profile and this can be described within the inertial sublayer by using a roughness length and a zero plane displacement, as illustrated in Fig. 4. Two main methods have been proposed for these parameters: the geometric method that uses parameters, which broadly describe the geometric form, or micrometeorological methods which use observations of wind and turbulence to derive parameters from the wind profile. The latter method requires tall towers and instrumentation. The former method requires knowledge of certain average geometric factors describing urban roughness elements, such as the average height of roughness elements (buildings or trees), fractional plan area, fractional frontal area, etc. Relationships can be derived from idealised flows over simplified arrays in wind tunnels, but need to be tested in real situations. One choice of relationship may not apply universally (Grimmond and Oke, 1999), but they may be of use in characterising urban areas for the urban pollution calculations routinely needed by air quality management planners, when the uncertainty in other factors may be greater. A classification of effective terrain roughness is often valuable in working situations (Davenport et al., 2000). It should be possible to associate aerodynamic parameters with each of the urban meteorological sites identified in the COST 715 inventory of urban meteorological stations (see COST 715 Final Report, Fisher et al., 2005).

The air quality modeller or planner needs to know a number of other key parameters. Some are familiar in the rural boundary layer, such as the surface heat flux and boundary layer height, but need modification in the urban boundary layer.

The blending height is a useful simplification above which the effective friction velocity and roughness length for a surface with inhomogeneous surface characteristics (spatially varying roughness or surface heat flux) can be defined. Associated with the blending height are aggregation formulae, or weighted averages, of the surface characteristics. For the regional heat flux a simple weighted average over the sub-areas of patches with different characteristics is used (Gryning and Batchvarova, 2001). For the effective roughness length a more complex averaging is needed. Such methods need to be tested, but are clearly essential for meso-scale models, in which surface characteristics are averaged over some grid square, within which some effective exchange of heat or momentum is visualised to take place. In such models the blending height for the given surface characteristics ought to correspond with the lowest grid level in the model. Within an urban area the inertial and roughness sublayers may only be well defined in parts of the urban boundary layer. It is only in these parts that the treatment of wind speed and turbulence described later in Sect. 6 applies.

In weak wind conditions, the influence of strong variations of surface characteristics may not be confined to a shallow fraction of the boundary layer, limiting the value of the blending height approach. When applicable, the blended surface heat flux is the appropriate quantity to use in estimating the height of the urban boundary layer (Baklanov, 2001a) and for the convective urban boundary layer simple slab models were found to perform quite well The literature is full of formulae (e.g. Baklanov, 2001b, for mixing height under stable conditions) and the air pollution modeller needs to apply these with care. 
There are other features of urban areas for which there are at present few practical formulae. For example the relationship between the wind speed at a standard $10 \mathrm{~m}$ height outside a city to the wind speed on a mast within the city is uncertain. For practical use, advice needs to be given on urban correction factors. The starting point for this has been the inventory of urban meteorological stations, which now comprises over 300 sites from all countries active in COST 715 (Müller et al., 2002). In the following section a practical method for obtaining appropriate meteorological data for undertaking urban air pollution calculations is discussed.

\section{Example of scheme for estimating wind speed and tur- bulence in the urban roughness sublayer using obser- vations at other sites}

As a working hypothesis, an urban reference height, $z_{\text {refu }}$, similar to that specified in the World Meteorological Organisation guide for rural sites $\left(z_{\text {refu }}=10 \mathrm{~m}\right)$ will be used. It is suggested that $z_{\text {refu }}=d+10 \mathrm{~m}$, where $d$ is the zero plane displacement. This suggestion is based on its similarity to the WMO guideline for siting of instruments in rural areas. However, it should be noted that for very large roughness elements (roughly higher than about $40 \mathrm{~m}$ ) this reference height would lie below mean roof level. Alternative formulations might therefore have to be considered. The scheme described below to estimate wind speed at a reference level (if available only from any other level) is not affected by this choice.

The procedure for estimating the wind speed at $z_{\text {refu }}$ from an observation at another height is the following.

Step 1 Roughness sublayer height and zero plane displacement

For use in later expressions, the height of the roughness sublayer, $z^{*}$, and the zero plane displacement, $d$, for the site under consideration have to be known. If they are not known from other, independent methods, the following methods can be used:

\section{Height of the roughness sublayer $z^{*}$}

This is a very poorly defined parameter and has not been subject to much investigation. Grimmond and Oke (1999) cite a number of estimates in the context of urban studies. They are all in the range given by Raupach et al. (1991), namely

$z^{*}=2 z_{H} t o 5 z_{H}$

where $z_{H}$ denotes the average building (or rather roughness element) height. Here it is suggested that the lower limit (i.e. $z^{*}=2 z_{H}$ ) is used for typical European cities for the following reasons:
1. $z^{*}$ can be interpreted as the height of the maximum Reynolds stress (see step 2$)^{2}$. The available full-scale data suggest that the maximum Reynolds stress occurs in the range $1.5 z_{H}<z<2.5 z_{H}$.

2. In wind tunnel studies, the maximum Reynolds stress is observed around $z=2 z_{H}$ (Rafailidis, 1997, for a regular array with flat roofs) and sometimes significantly lower (see Kastner-Klein and Rotach, 2004, for a "real" array for Nantes, and Rafailidis, 1997, for a regular array with slanting roofs).

3. Using the suggested concept of $z^{*}=2 z_{H}$ to simulate urban tracer dispersion experiments (Rotach, 2001), yields the best agreement between observations and modelled results.

However, if the density of roughness elements is very low, one of the other expressions as presented by Grimmond and Oke (1999) may be considered.

\section{Zero plane displacement $d$}

If $d$ is not known from other independent methods, it may be estimated using the distribution and density of buildings (roughness elements). A comprehensive collection of methods can be found in Grimmond and Oke (1999). The simplest of the methods studied relates the zero plane displacement to the average building height: $d=0.7 z_{H}$. Grimmond and Oke's (1999) results indicate that this simple method yields reasonable results for $0.3 \leq \lambda_{P} \leq 0.5$ and $0.1 \leq \lambda_{F} \leq 0$. Here the two parameters used are the non-dimensional plan area, $\lambda_{P}=A_{P} / A_{T}$ and the nondimensional frontal area, $\lambda_{F}=A_{F} / A_{T}$, where $A_{T}$ denotes the total area, $A_{P}$ is the area occupied by buildings, and $A_{F}$ is the frontal area of the buildings (calculated as the product of the average height and the average width).

\section{Step 2 Estimating the friction velocity}

The basis of this step is a postulated variation of the Reynolds stress with height within the roughness sublayer. The suggested parameterisation is (Rotach, 2001)

$$
\left(\frac{u_{* l}}{u_{* I S}}\right)^{b}=\sin \left(\frac{\pi}{2} Z\right)^{a} \text {, when } Z \leq 1
$$

where $u_{* l}(z)$ is the local scaling velocity, $u_{* l}^{2}(z)=-\overline{u^{\prime} w^{\prime}(z)}$, $u_{* I S}$ is the friction velocity (evaluated in the inertial sublayer) and $Z=(z-d) /\left(z^{*}-d\right)$ is a non-dimensional height.

Note that this parameterisation assumes that the height of the roughness sublayer, $z^{*}$, corresponds to the height where the maximum (absolute value) of Reynolds stress occurs. This maximum value of Reynolds stress is used to derive the

\footnotetext{
${ }^{2}$ Rather than the height where the influence of individual roughness elements on mean and turbulence profiles vanishes
} 


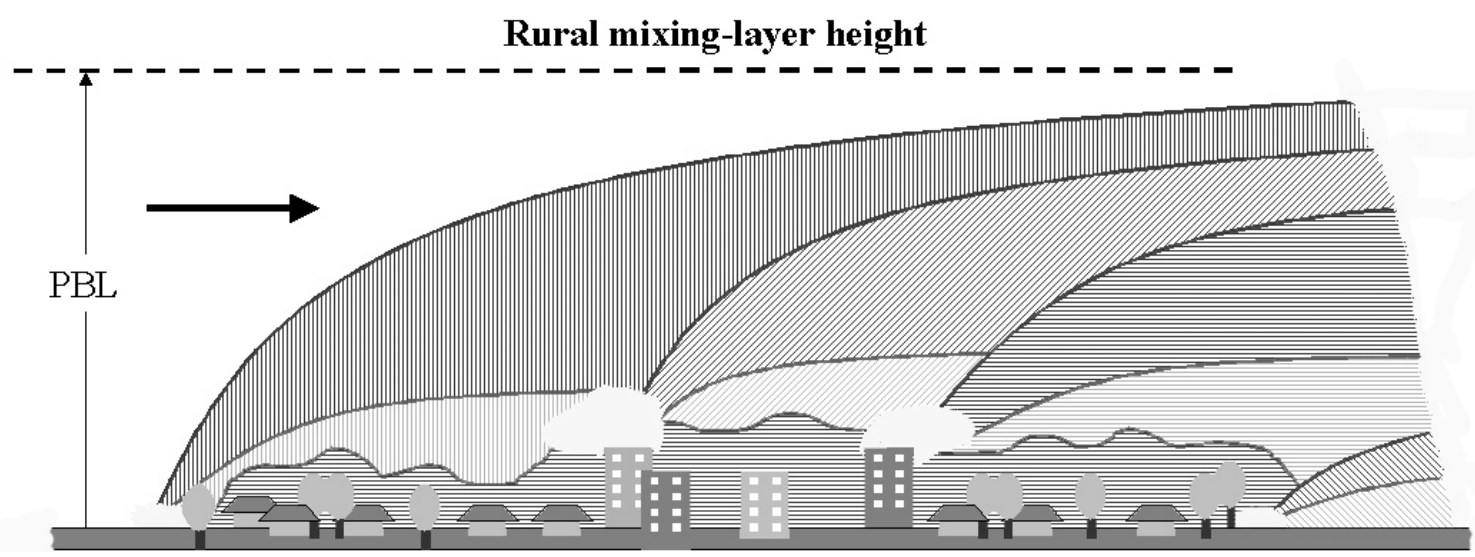

Fig. 5. Schematic figure showing large scale features of boundary layer over an urban area. The upper plumes represent the urban internal boundary layers where advection processes are important. The inertial layers lie between the internal boundary layers and the roughness sublayer next to the ground, and are in equilibrium with the underlying surface where Monin-Obukhov scaling applies. The roughness sublayers are the lowest layers next to the ground and are highly inhomogeneous both in their vertical and horizontal structure. In addition there are zones which cannot be classified in any of the three types, where adjustment between neighbourhoods takes place, with large accelerations and shear in the flow near the top of the canopy (after Gryning). PBL is the planetary boundary layer.

friction velocity of the flow "far away from the surface" i.e. within the inertial sublayer. It is denoted $u_{* I S}$.

1. If a measurement of Reynolds stress is available with a measurement height below $z^{*}$ the next step is to estimate $u_{* I S}$ using Eq. (3).

2. If a measurement of Reynolds stress is available at $z>z *$ and the measurement height is not much larger than $z^{*}$, the observation may be interpreted as $u_{* I S}$.

3. A measurement of Reynolds stress is available, but outside the city, then $u_{* I S}$ may be estimated from (Bottema, 1995)

$\frac{u_{* 1}}{u_{* 2}}=\left(\frac{z_{01}}{z_{02}}\right)^{\alpha}$

where $\alpha$ is a parameter that can be taken to equal $0.0706, u_{* 1}$ corresponds to the urban friction velocity $\left(=u_{* I S}\right)$ and $u_{* 2}$ is the rural friction velocity and $z_{01}$ and $z_{02}$ the corresponding roughness lengths.

Step 3 Estimation of the wind speed $\bar{u}$ at $z_{\text {refu }}=d+10 \mathrm{~m}$

Within the roughness sublayer the non-dimensional gradient for wind (using Monin-Obukhov Similarity Theory) can be used (Rotach, 1993) provided that the local scaling velocity, $u_{* l}(z)$, is used rather than the friction velocity which corresponds to $u_{* I S}$. Thus

$\frac{d \bar{u}}{d z} \frac{\kappa z^{\prime}}{u_{* l}\left(z^{\prime}\right)}=\phi_{m}\left(z^{\prime} / L_{l}\left(z^{\prime}\right)=\left(1-19.3 \frac{z^{\prime}}{L_{1}\left(z^{\prime}\right)}\right)\right.$

for unstable conditions, and

$\frac{d \bar{u}}{d z} \frac{\kappa z^{\prime}}{u_{* l}\left(z^{\prime}\right)}=\phi_{m}\left(z^{\prime} / L_{l}\left(z^{\prime}\right)=\left(1+6 \frac{z^{\prime}}{L_{1}\left(z^{\prime}\right)}\right)\right.$ for stable stratification, where $z^{\prime}=z-d$. Note that Eqs. (5) and (6) are based not only on a local scaling velocity but also on a local Monin-Obukhov length

$L_{l}\left(z^{\prime}\right)=\frac{-\bar{\theta} u_{* l}^{2}}{\kappa g \overline{w^{\prime} \theta^{\prime}}}$

where $\theta$ is the mean potential temperature, $\kappa$ is the von Karman constant $(\kappa=0.4)$ and $g$ is the acceleration due to gravity. The turbulent heat flux $\overline{\mathrm{w}^{\prime} \theta^{\prime}}$ is assumed to be constant in the absence of more detailed information and can be derived from the surface energy balance (see Sect. 4). Integrating Eqs. (4) or (5) numerically either down or up, the mean wind speed at a required level can be found from an observation at any height within the roughness sublayer. If the observation stems from above the roughness sublayer (but below $10 \%$ of the boundary layer height), Eqs. (5) and (6) can be used with $u_{* I S}$ rather than $u_{* l}(z)$ down to $z^{*}$, and from there the procedure described above can be used.

The approach above has been tested within COST 715 activities and appears to work reasonably well for the few locations where detailed measurements are available (Fisher et al., 2005). However, more testing is necessary. It must be emphasised that the turbulence profiles suggested are intended to produce area averaged profiles, with an average scale of several times the dimensions of one building, rather than to describe the profile at one location. This is consistent with urban dispersion calculations which are generally tested in an integrated sense, by looking at dispersion of a tracer over moderate distances rather than just very locally.

However simple, conceptual approaches need to be combined with more complex models to provide insight. As seen from Fig. 5 the simplifications only apply to parts of the urban boundary layer and transition zones exist where 


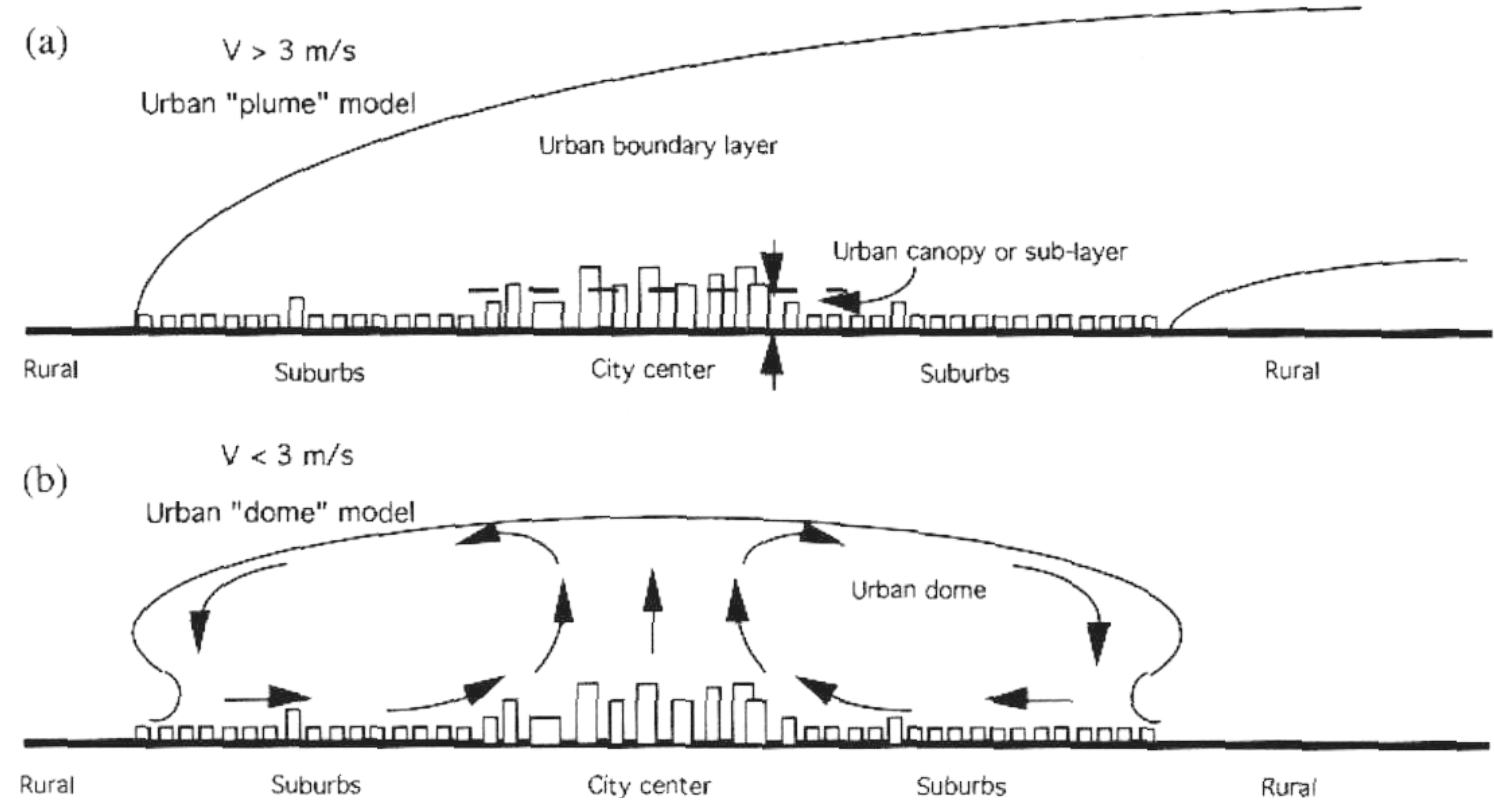

Fig. 6. Meso-scale features which may be present within an urban area. These show possible effects of advection which complicate considerably the "simple" picture shown in earlier figures (after Janour).

the methods would not be applicable. In addition the urban boundary layer may be subject to meso-scale features (see the organised flows in Fig. 6), further complicating any description and requiring the use of meso-scale transport models.

\section{Conclusions}

In the framework of COST 715 a number of concepts have been reviewed and tested that allow an appropriate description of meteorological parameters necessary for dispersion modelling in urban areas. In particular the importance of introducing a roughness sublayer in the lowest part of the urban boundary layer has been highlighted. The roughness sublayer, although not unique to urban areas, is of great importance due to its vertical extension over large roughness elements. The concepts as reviewed in the present paper offer a more realistic description of flow and energy balance components for urban dispersion studies. In current practice, however, many "urban dispersion models" still have "surface layer characteristics" (i.e. Monin-Obukhov Similarity Theory) adjacent to the surface with the exception of possibly adjusting the roughness length and setting some limits to the Monin-Obukhov length to avoid very stable conditions in urban areas (reviewed by Craig and Bornstein, 2002).

At present in the language of the NUSAP criteria, the pedigree of Monin-Obukhov similarity theory is high and modellers may be reluctant to alter an established approach. In terms of assessment, the ideas developed under COST 715 and elsewhere, try to capture some of the real processes within the urban boundary layer. Only future research will establish their general validity. In particular, the concepts presented are based to a large degree on the typical structure of European cities and need to be extended to suburban surfaces and typical structures over cities in other continents. Moreover simple, conceptual approaches need to be combined with more complex models to provide insight. A complete summary of results from COST 715 can be found in the final report (Fisher et al., 2005).

\section{Appendix}

Work within COST 715 was undertaken in four Working Groups namely:

The dynamics of the urban boundary layer, Working Group 1, Chairman M. Rotach, Swiss Federal Office for Meteorology and Climatology, MeteoSwiss,

The thermal structure of the urban surface and the boundary layer height, Working Group 2, Chairman M. Piringer, Central Institute for Meteorology and Geodynamics, Vienna,

Air pollution episodes, Working Group 3, Chairman J. Kukkonen, Finnish Meteorological Institute

Preparation of meteorological input data for urban air pollution models, Working Group 4, Chairman M. Schatzmann, University of Hamburg, Meteorological Institute. 
A series of COST 715 reports have been published by the Luxembourg Office for Official Publications of the European Communities:

1. Surface energy balance in urban areas. Extended abstracts of an expert meeting, Antwerp 12 April 2000, COST report EUR 19447.

2. Preparation of meteorological input data for urban site studies. Proceedings of the workshop, 12 June 2000, Prague, COST report EUR 19446.

3. Meteorology during peak pollution episodes. Working Group 3 status report, COST report EUR 19448.

4. Workshop on urban boundary layer parameterisations. Extended abstracts, Zurich 24-25 May 2001, COST report EUR 20355.

5. Mixing height and inversions in urban areas. Proceedings of the Workshop, 3-4 October 2001, Toulouse, COST report EUR 20451.

6. The urban surface energy budget and mixing height in European cities: data, models and challenges for urban meteorology and air quality. Demetra, Bulgaria, ISBN 954-9526-29-1. COST 715 Working Group 2 Final Report, 2005.

Web addresses of the Working Groups are:

\section{COST715}

http://www.dmu.dk/atmosphericenvironment/cost715.htm

Working Group 1

http://www.iac.ethz.ch/en/research/cost715/cost715_2.html

Working Group 2

http://cost/fmi.fi/wg2/

Working Group 3

http://cost/fmi.fi

Working Group 4

http://www.mi.uni-hamburg.de/cost715/form.html

Urban Meteorological Station Survey

http://www.mi.uni-hamburg.de/cost715/inventory.html

Edited by: S. M. Joffre

\section{References}

Baklanov, A.: The mixing height in urban areas - a review, COST 715 Expert Workshop "Mixing Height and Inversions in Urban Areas", Toulouse, France, 3-4 October, 2001a.

Baklanov, A.: Parameterisation of SBL height in atmospheric pollution models, paper at 25th NATO/CCMS International Technical Meeting on Air Pollution Modelling and its Application, Louvain-la-Neuve, Belgium, 2001b.

Bottema, M.: Aerodynamic roughness parameters for homogeneous building groups, report Sub-meso 18, Lab. de Mécanique des Fluides, Ecole Centrale de Nantes, 1995.

Christen, A. and Vogt, R.: Energy and radiation balance of a central European city, Int. J. Climatol., 24, 1395-1421, 2004.

Coceal, O. and Belcher, S. E.: Mean winds through an inhomogeneous urban canopy, Boundary Layer Meteorol., 115, 47-68, 2005.

Craig, K. J. and Bornstein, R. D.: Urbanisation of numerical mesoscale models, in: Workshop on Urban Boundary Layer Parameterisations, edited by: Rotach, M. W., Fisher, B., and Piringer, M., EUR 20355, (ISBN: 92-894-4143-7), 17-30, 2002.

Davenport, A. G., Grimmond, C. S. B., Oke, T. R., and Wieringa J.: Estimating the roughness of cities and sheltered country, American Meteorological Society, 12th Conference on Applied Climatology, Boston, USA, 2000.

Fisher, B., Joffre, S., Kukkonen, J., Piringer, M., Rotach, M. W., and Schatzmann, M. (Eds.): Meteorology applied to Urban Air Pollution Problems, Final Report COST Action 715, Demetra, Bulgaria, ISBN 954-9526-30-5, 2005.

Grimmond, C. S. B. and Oke, T. R.: Aerodynamic properties of urban areas derived from the analysis of surface form, J. Appl. Meteorol., 38, 1262-1292, 1999.

Grimmond, C. S. B. and Oke, T. R.: Turbulent heat fluxes in urban areas: observations and local-scale urban meteorological parameterisation scheme (LUMPS), J. Appl. Meteorol., 41, 792-810, 2002.

Gryning, S.-E. and Batchvarova, E.: Pre-processor for regional scale fluxes and mixed height over inhomogeneous terrain, paper at 25th NATO/CCMS International Technical meeting on Air Pollution Modelling and its Application, Louvain-la-Neuve, Belgium, 2001.

de Haan, P. and Rotach, M. W.: A novel approach to atmospheric dispersion modelling: The puff-particle model (PPM), Quart. J. Roy. Meteorol. Soc., 124, 2771-2792, 1998.

Karppinen, A., Joffre, S. M., and Kukkonen, J.: The refinement of a meteorological preprocessor for the urban environment, Int. J. Environ. and Pollution, 14, 1-9, 2000a.

Karppinen, A., Kukkonen, J., Elolähde, T., Konttinen, M., Koskentalo, T., and Rantakrans, E.: A modelling system for predicting urban air pollution: model description and applications in the Helsinki metropolitan area, Atmos. Environ., 34, 3723-3733, $2000 b$.

Kukkonen, J., Pohjola, M., Sokhi, R. S., Luhana, L., Kitwiroon, N., Rantamäki, M., Berge, E., Odegaard, V., Slørdal, L.H., Denby, B., and Finardi, S.: Analysis and evaluation of selected local-scale $\mathrm{PM}_{10}$ air pollution episodes in four European cities: Helsinki, London, Milan and Oslo, Atmos. Environ., 39, 27592773, 2005.

Kastner-Klein, P. and Rotach, M. W.: Mean flow and turbulence characteristics in an urban roughness sublayer, Boundary Layer 
Meteorol., 111, 55-84, 2004.

Martilli, A., Clappier, A., and Rotach, M. W.: An urban surface exchange parameterisation for mesoscale models, Boundary Layer Meteorol., 104, 261-304, 2002.

Middleton, D. R.: Meteorological challenges in urban air pollution, Clean Air, 32, 14-19, 2002.

Müller, W. J., Heits, B., and Schatzmann, M.: A prototype station for the collection of urban meteorological data, Proceedings 8th International Conference on Harmonisation within Atmospheric Dispersion Modelling for Regulatory Purposes, Sofia, Bulgaria, 14-17 October 2002.

Piringer, M., Grimmond, C. S. B., Joffre, S. M., Mestayer, P., Middleton, D. R., Rotach, M. W., Baklanov, A., De Ridder, K., Ferreira, J., Guilloteau, E., Karppinen, A., Martilli, A., Masson, V., Oke, T. R., and Tombrou, M.: Investigating the surface energy balance in urban areas - recent advances and future needs, Water, Air and Soil Poll. Focus, 2, 1-16, 2002.

Piringer, M. and Joffre, S. (Eds.): The urban surface energy budget and mixing height in European cities: Data, models and challenges for urban meteorology and air quality, Demetra, Bulgaria, ISBN 954-9526-29-1, 2005.

Rafailidis, S.: Influence of building areal density and roof shape on the wind characteristics above a town, Boundary Layer Meteorol., 85, 255-271, 1997.
Rantamäki, M., Pohjola, M. A., Tisler, P., Bremer, P., Kukkonen, J., and Karppinen, A.: Evaluation of two versions of the HIRLAM numerical weather prediction model during an air pollution episode in southern Finland, Atmos. Environ., 39, 27752786, 2005.

Raupach, M. R., Antonia, R. A., and Rajagopalan, S.: Rough-wall turbulent boundary layers, Appl. Mech. Rev., 44, 1-25, 1991.

Rotach, M. W.: Turbulence close to a rough urban surface, Part II: Variances and gradients, Boundary Layer Meteorol., 66, 75-92, 1993.

Rotach, M. W.: Simulation of urban-scale dispersion using a Lagrangian stochastic dispersion model, Boundary Layer Meteorol., 99, 379-410, 2001.

Schatzmann, M., Brechler, J., and Fisher, B. (Eds.): Preparation of meteorological input data for urban site studies, COST European co-operation in the field of scientific and technical research, Luxembourg Office for Official Publications of the European Communities, EUR 19446, 2001.

Soulhac, L., Carissimo, B., Mejean, P., Perkins, R. J., and Van Liefferinge, R.: A theoretical and experimental study of turbulent flow in the urban canopy, 8th International Conference on Harmonisation within Atmospheric Dispersion Modelling for Regulatory Purposes, Sofia, Bulgaria, 14-17 October 2002. 